LNS-98-277

UR-1516 ER/4056/911

FNAL-CONF-98/119

\title{
A Small Target Neutrino Deep-Inelastic Scattering Experiment at the First Muon Collider
}

\author{
Deborah A. Harris* and Kevin S. McFarland ${ }^{\dagger}$ \\ * University of Rochester, Rochester, NY 14627 \\ ${ }^{\dagger}$ Massachusetts Institute of Technology, 77 Massachusetts Ave., Cambridge, MA 02139
}

\begin{abstract}
Several different scenarios for neutrino scattering experiments using a neutrino beam from the muon collider complex are discussed. The physics reach of a neutrino experiment at the front end of a muon collider is shown to extend far beyond that of current neutrino experiments, since the high intensity neutrino beams one would see at the muon collider allow for a large flexibility in choosing neutrino targets. Measurements of quark spin, A-dependence of the structure function $x F_{3}$ and neutral current chiral couplings to quarks are outlined.
\end{abstract}

\section{INTRODUCTION}

Neutrino deep-inelastic scattering has proven an invaluable tool to study hadron structure, QCD, and the electroweak force. Neutrinos give the only clean measurement of the valence quark distribution inside a nucleon, and have a very distinct signature for scattering off a strange quark inside the nucleon sea. Neutrino-nucleon scattering also provides two important tests of QCD, through structure function evolution and the Gross Llewellyn Smith Sum Rule. Both tests can also provide precise measurements of the strong coupling constant, $\alpha_{s}$ [1]. Neutrino scattering experiments which can reconstruct neutral current interactions also provide a stringent test of electroweak symmetry breaking.

In the past, neutrino experiments have been shaped by the low neutrino scattering cross section. To get ample statistics experiments have had to use massive active targets. For example, the $\mathrm{CCFR} / \mathrm{NuTeV}$ experiments used a target of 700 tons of steel instrumented with scintillators [2], and the NOMAD experiment used a target of 2.7 tons of drift chambers [3]. In reference [4] the results from a simulation of the neutrino beams resulting from high energy muon beams were given, and event samples of comparable statistics are attainable using targets of only several $\mathrm{g} / \mathrm{cm}^{2}$ thickness. In this paper we consider targets that have previously been used for muon scattering, and discuss the novel physics that suddenly becomes feasible when placing these targets in extremely intense neutrino beams. 


\section{NEUTRINO SCATTERING KINEMATICS}

By including known $\nu$-nucleon differential cross sections in the Monte Carlo described in reference [4], one can predict the kinematic reach of a year's worth of data per $\mathrm{g} / \mathrm{cm}^{2}$ of target. It is important to note that since the neutrino beams from a muon collider are not more energetic than conventional beams, the reach in momentum transfer squared $\left(Q^{2}\right)$ of these experiments is comparable to what has already been measured. However, since $\alpha_{s}$ is large and changing rapidly in the region $1<Q^{2}<100 \mathrm{GeV}^{2}$, this is precisely where one wants to make perturbative QCD measurements. The kinematic variable one could extend in reach is $x$, or the struck quark's fractional momentum. To probe smaller and larger $x$ regions one needs both high statistics and good detector acceptance and resolution. To maximize statistics one could use a target which would result in a optimal number of interactions (for example, .05) per turn. This target would still be rather small, and would not degrade the event reconstruction, compared to previous neutrino experiments. The remainder of the experiment could then be a low-mass spectrometer with some particle identification, modeled on non-neutrino fixed target experiments (see reference [4]). A target of the size described above with a low mass detector to reconstruct the scatters would result in about $2 \times 10^{7}$ events per year for one of the recirculating linacs, and a factor of 100 more than that for an experiment downstream of a collider ring's straight section. Figure 1 shows the number of events expected per $\mathrm{g} / \mathrm{cm}^{2}$ as a function of $x$ and $Q^{2}$, assuming a $20 \mathrm{~cm}$ radius target. The reach in $x$ for a "high statistics target" in a given $Q^{2}$ region is an order of magnitude beyond what is currently measured. As there have been surprises in the low $x$ region, for example, the fast rise of $F_{2}$ as seen by HERA [5], the question arises, is the valence quark distribution well-behaved at low $x$, or does something unexpected happen there too?

\section{Strong Coupling Constant Determinations}

Neutrino deep-inelastic scattering provides two clean measurements of the strong coupling constant $\alpha_{s}$ : one from the $Q^{2}$ dependence of the non-singlet structure function $x F_{3}$, and one from the Gross Llewellyn Smith Sum Rule. Both of these measurements are independent of the gluon distribution, and the latter has very small perturbative QCD uncertainties. Currently, however, both measurements are limited by experimental systematic uncertainties: the $x F_{3}$ evolution is limited by the uncertainty in the energy calibration [2], and the GLS Sum Rule is limited by the uncertainty in both the overall neutrino cross section, and the ratio of neutrino to anti-neutrino cross sections [6]. Furthermore, both cross section measurements are themselves limited by systematic uncertainties [7]. A neutrino experiment at a muon collider could address all three of these issues in fundamentally different ways, because of the way the beam is formed.

Neutrinos from a monochromatic beam of muons have a very distinct spectrum 

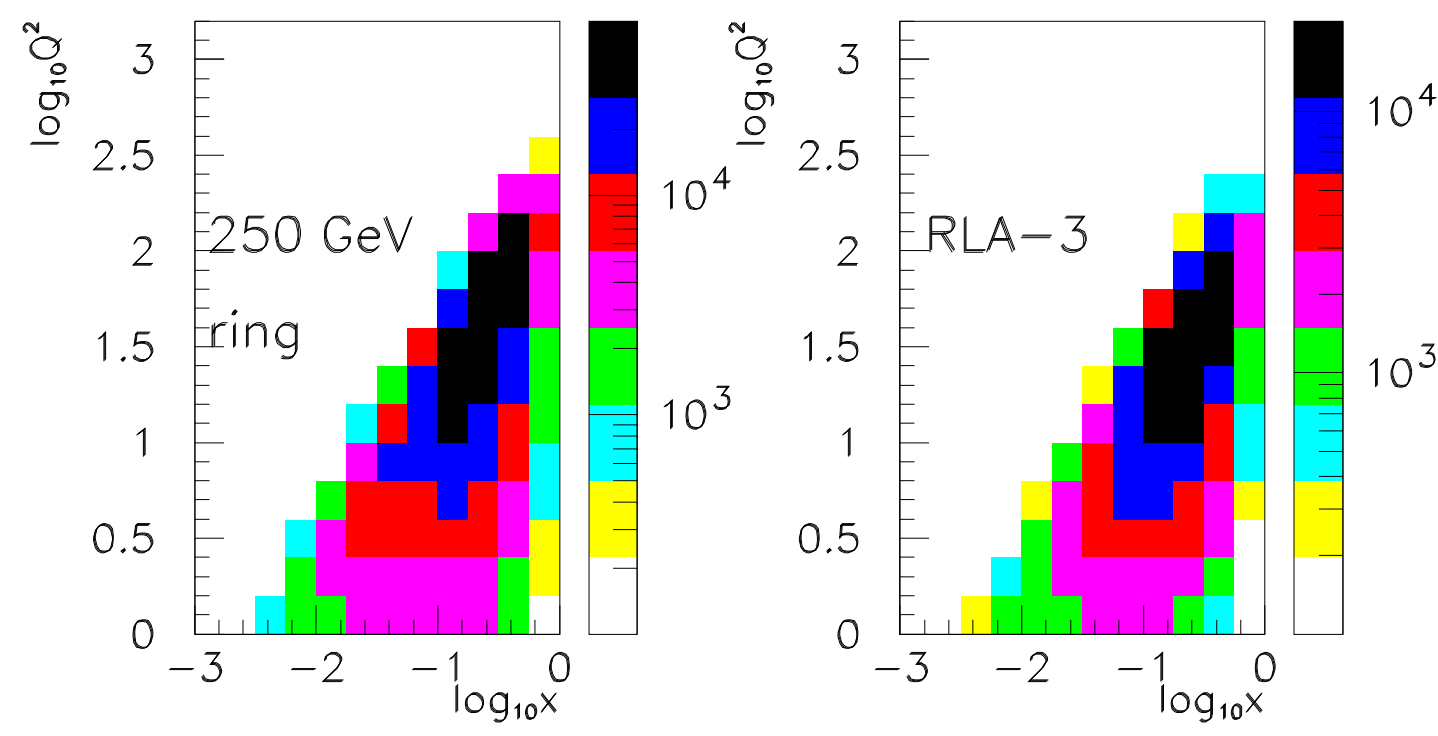

FIGURE 1. $x$ versus $Q^{2}$ distributions for two possible neutrino experiments. Units are in events per year per $\mathrm{g} / \mathrm{cm}^{2}$ of target for a $20 \mathrm{~cm}$ radius target.

peaked towards the high end of the available energy from the muon. Muon decay itself is very well understood, and the muon energies at the collider are predicted to be measurable to a few parts per million [8]. By calibrating the detector to the end point of the neutrino spectrum one should be able to achieve much better than the current energy scale uncertainty in neutrino experiments, which is about $1 \%$ $[2]$.

Similarly, the muon currents in the beam-lines can be measured, and given the energy of the muons and the currents and the geometry of the beam-line, the neutrino flux at a given detector can be accurately predicted. The availability of an independent flux measurement for a neutrino experiment will be an important improvement and will dramatically change the nature of absolute neutrino cross section measurements, as well as the scale of the uncertainties in those measurements.

\section{LIGHT TARGETS}

Large low-Z targets have already been used successfully by muon scattering experiments. The sizes of liquid hydrogen targets are in general limited by safety considerations, and a liquid hydrogen target of $1 \mathrm{~m}$ thickness and radius approximately $10 \mathrm{~cm}$ has been used by experiment E665 at Fermilab [9]. A target of this volume of hydrogen with a $20 \mathrm{~cm}$ radius would have a thickness of $1.75 \mathrm{~g} / \mathrm{cm}^{2}$, resulting in data samples of slightly over a million $\nu$ charged current events per year. The per turn event rate on this target would still be small enough so that pile-up 


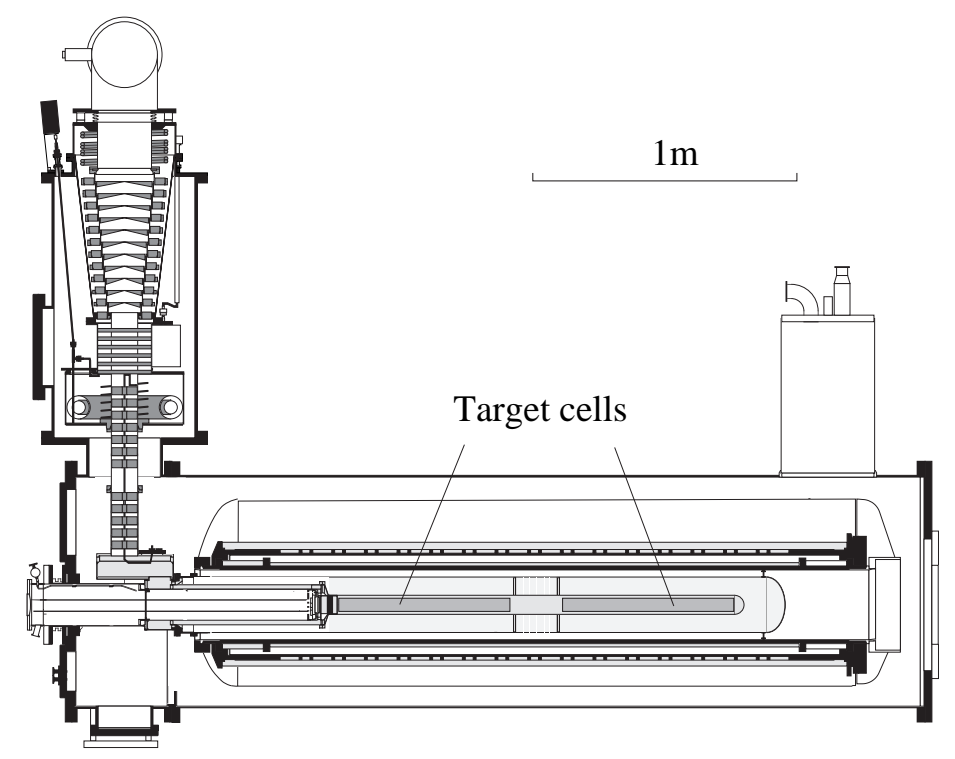

\section{Refrigerator Superconducting magnets}

FIGURE 2. Polarized butanol target used in the SMC experiment (see text). The total target mass was $3 \mathrm{~kg}$, and the polarized fraction of the target was approximately $10 \%$.

in the target itself would not be a problem.

\section{NUCLEAR EFFECTS}

Charged lepton experiments have studied nuclear dependences of the structure function $F_{2}$ and have seen a sizeable effect [10] [9], yet no nuclear dependences have been seen in $x F_{3}$, and for now theory must only assume that the integral of the valence quark distribution is independent of nuclear corrections. By scattering neutrinos off several light targets one could finally determine whether or not valence quarks know anything about what kind of nucleus they are in. If one had both a hydrogen and a deuterium target, one could measure if $u(x)$ in the proton is indeed equal to $d(x)$ in the neutron. Such a measurement would either confirm or challenge our assumptions about isospin symmetry. The effects seen in charged lepton scattering are large; for example, $2 F_{2}^{d} / F_{2}^{p}-1$ is $0.935 \pm 0.008$ (stat) \pm 0.034 (syst) at $x$ below 0.01 [9], and at higher $x$ this ratio is even farther away from unity. With hydrogen and deuterium targets of the sizes suggested above one would have enough statistics to measure the quantity $2 x F_{3}^{d} / x F_{3}^{p}-1$ as a function of $x$ to better than a per cent, over a reasonably large $x$ range. 


\section{POLARIZED TARGETS}

One exciting possibility in such a light-target neutrino experiment is measuring neutrino scattering from polarized targets. The neutrino beams from the muon collider can produce on the order of $5 \times 10^{5}$ events $/ \mathrm{kg} /$ year in a target; therefore, a target with approximately $20 \mathrm{~kg}$ of polarized protons would produce excellent statistics.

An example of a large polarized target used in the past is the SMC solid butanol target [11] (Figure 2), which was a $2.5 \mathrm{~cm}$ radius cylinder containing $0.3 \mathrm{~kg}$ of polarized protons. If such a target could be scaled up to the size of the neutrino beam, approximately $20 \mathrm{~cm}$ in radius, this would provide sufficient mass for a high statistics neutrino experiment.

\section{SPIN PHYSICS}

If the polarized target described above were feasible, the physics motivations would be many-fold. Deep-inelastic scattering from hydrogen targets would probe the following processes

$$
\begin{array}{ll}
\nu u \rightarrow \mu^{-} d & \nu \bar{d} \rightarrow \mu^{-} \bar{u} \\
\bar{\nu} d \rightarrow \mu^{+} u & \overline{\nu u} \rightarrow \mu^{+} \bar{d} \\
\bar{\nu} s \rightarrow \mu^{+} c & \nu \bar{s} \rightarrow \mu^{-} \bar{c},
\end{array}
$$

and their Cabbibo-suppressed analogs. The beauty of probing polarized targets with neutrinos is that these six processes are clearly separable. $y$ distributions can be used to separate quark and anti-quark targets; neutrinos and anti-neutrinos each pick one flavor of a quark doublet; strange quarks can be tagged by final state charm in its decay to high momentum leptons or via its finite lifetime.

This leads to the possibility of a measurement of the polarization of the proton's quarks by flavor, with sea and valence contributions separated. This measurement would be relatively independent of details of final state hadronization in contrast with current experimental programs of measuring the polarization of the strange sea which rely on final state strange mesons or baryons.

The statistical challenge for such an experiment would be the measurement of the strange polarization from final state di-lepton events, where one lepton comes from the decay of charm. The total di-lepton cross-section is approximately $2 \%$, therefore, on a $200 \mathrm{~kg}$ butanol target, approximately $1 \times 10^{6}$ neutrino and $5 \times 10^{5}$ anti-neutrino di-lepton events per year would be observed, about half of which come from scattering off of strange quarks. With these statistics, a raw asymmetry could be measured with a $1.5 \times 10^{-3}$ precision. Assuming the $d$ quark asymmetry would be better known from the more common processes listed above, this would translate into a precision in the strange sea polarization of $3 \%$ in a one year run.

This precision is reasonable for testing models for the spin of the strange sea. A recent prediction for the strange sea polarization in an effective chiral quark model [12] estimates a $-8 \%$ polarization. 


\section{NEUTRAL CURRENT QUARK COUPLINGS}

Neutrino deep-inelastic scattering from light, non-isoscalar nuclei also gives the possibility of doing precision measurements of the left- and right-handed couplings of the neutral current to quarks. To date, precision neutrino and atomic physics experiments have probed primarily heavy nuclei and thus could only measure isoscalar combinations of couplings. Such observations could determine whether the lingering evidence for some enhanced coupling of the neutral current to $b$-type quarks [13] is only observed in the $b$ system or whether it is perhaps the first evidence of some generation-independent phenomenon.

\section{CONCLUSIONS}

We have presented several different physics motivations for a deep-inelastic scattering neutrino experiment at the front end of the muon collider complex. The possibility of scattering neutrinos off either light elements or polarized targets will address a wide range of issues in our understanding of hadron structure. The experience from polarized targets used in muon scattering experiments will be particularly useful, as it would enable measurements of the individual quark contributions to the spin of the proton. In order to best take advantage of the neutrino beams at the muon collider complex a run of two or more years using several different cryogenic targets would be optimal. Furthermore, the characteristics of this new neutrino beam would be ideal for new precision measurements of both the strong coupling constant, and the neutral current quark couplings. In summary, deepinelastic scattering neutrino experiments at the muon collider have the potential to fundamentally change our understanding of the baryons that make up most of the observed universe.

\section{REFERENCES}

1. D.J. Gross and C.H. Llewellyn Smith, Nucl. Phys. B14 337 (1969), and G. Altarelli and G. Parisi, Nucl. Phys. B126 298 (1977).

2. W.G. Seligman et al., Phys. Rev. Lett. 79, 1213 (1997).

3. M. Laveder, Nucl. Phys. Proc. Suppl. 48, 188 (1996).

4. D.A. Harris and K.S. McFarland, "Detectors for Neutrino Physics at the First Muon Collider", these proceedings.

5. J. Breitweg et al., Phys. Lett. B407, 432 (1997).

6. J. Yu et al., proceedings to XXXI Rencontres de Moriond, QCD and Hadronic Interactions (1997).

7. W.G. Seligman, Ph.D. Thesis, NEVIS-292 (1997).

8. R. Raja, A. Tollestrup, FERMILAB-Pub-97/402-E, submitted to Phys. Rev. D.

9. M.R. Adams et al.,Phys. Rev. Lett. 75,1466 (1995).

10. EMC, J.J. Aubert et al., Phys. Lett. 123B, 275 (1983) 
11. Adams, D., et al., Phys. Rev. D56, (1997)

12. E. Eichten, I. Hinchliffe, C. Quigg, Phys. Rev. D45, 2269 (1992).

13. Particle Data Group, Phys. Ref. D54, (1996). 